\title{
Toward quantum information processing by nuclear magnetic resonance: Pseudopure states and logical operations using selective pulses on an oriented spin $3 / 2$ nucleus
}

\author{
Neeraj Sinha and T. S. Mahesh \\ Department of Physics, Indian Institute of Science, Bangalore-560 012, India \\ K. V. Ramanathan \\ Sophisticated Instruments Facility, Indian Institute of Science, Bangalore-560 012, India \\ Anil Kumara) \\ Department of Physics and Sophisticated Instruments Facility, Indian Institute of Science, \\ Bangalore-560 012, India
}

\begin{abstract}
Nuclear magnetic resonance spectroscopy has demonstrated significant experimental progress toward the development of quantum computations. The developments so far have taken place mainly through the use of spin $\frac{1}{2}$ nuclei. In this paper we describe the use of a spin $\frac{3}{2}$ nucleus, oriented in a liquid crystal matrix for the creation of pseudopure states and the implementation of a complete set of two-qubit reversible logic gates using single-quantum transition-selective pulses, extending the range of practice of NMR toward quantum computation.
\end{abstract}

\section{INTRODUCTION}

One of the challenges of present day NMR quantum computing is to increase the number of qubits. ${ }^{1}$ Solution state NMR is limited by the size and number of throughbond resolved indirect spin-spin couplings. This limitation can be lifted by orienting molecules in liquid crystalline media yielding large intramolecular dipolar couplings. ${ }^{2,3}$ The number of coupled nuclei can be increased, since such systems are not limited by throughbond couplings. The quadrupolar nuclei in a liquid crystalline matrix have an added advantage that each nucleus can be treated as more than one qubit. ${ }^{4} \mathrm{~A}$ nucleus with a half-integral spin $I$ can be treated as an $N$ qubit system provided $(2 I+1)=2^{N}$, thereby increasing the number of qubits per nucleus. The Hamiltonian of a quadrupolar nucleus oriented in a liquid crystalline matrix, in the presence of a large $B_{0}$ field, having a first-order quadrupolar coupling, can be written as ${ }^{5}$

$$
\begin{aligned}
\mathcal{H} & =-\omega_{0} I_{z}+\frac{e^{2} q Q}{4 I(2 I-1)}\left(3 I_{z}^{2}-I^{2}\right) S \\
& =-\omega_{0} I_{z}+\Delta\left(3 I_{z}^{2}-I^{2}\right),
\end{aligned}
$$

where $\omega_{0}=\gamma B_{0}$ is the Larmor frequency, $S$ the order parameter at the site of the nucleus, and $e^{2} q Q$ is the quadrupolar coupling. If the order parameter $S$ is small, the effective quadrupolar coupling $\Delta$ can be of the order of a few $\mathrm{kHz}$ in spite of $e^{2} q Q$ being of the order of several MHz. Thus, orientation of quadrupolar nuclei by liquid crystals makes it possible to observe the satellite transitions due to first-order quadrupolar couplings within a few $\mathrm{kHz}$ range. However, the quadrupolar splitting thus obtained is large compared to the $J$ couplings

\footnotetext{
a) Author to whom correspondence should be addressed. Electronic mail: anilnmr@physics.iisc.ernet.in
}

among spin $\frac{1}{2}$ nuclei in an isotropic solution. Therefore short transition-selective pulses can be used, increasing the speed of NMR computation. ${ }^{3}$ The large frequency separation also enables the easy application of transition-selective pulses.

The creation of pseudopure states in a spin $\frac{3}{2}$ system oriented in a liquid crystalline matrix has recently been demonstrated by utilizing the nonlinear excitation of doublequantum transitions. ${ }^{4}$ In this paper we demonstrate, in such a system, the creation of pseudopure states and also the implementation of a complete set of two qubit gates by the use of transition-selective single-quantum pulses. The excitation of double-quantum coherence is achieved here through the use of a cascade of noncommuting transition-selective singlequantum pulses. ${ }^{6}$ The advantages of using single-quantum transition-selective pulse are (i) the ease of calibration of pulse lengths and powers, and (ii) the simple sequence of pulses for the implementation of various logical operations compared to the pulse sequences needed for the $J$-evolution technique. ${ }^{6-11}$ To the best of our knowledge this is the first implementation of a complete set of one-to-one logical gates on quadrupolar nuclei, oriented in a liquid crystalline matrix, except for an implicit C-NOT gate in Ref. 4.

\section{EXPERIMENT}

Experiments were carried out on a ${ }^{7} \mathrm{Li}$ nucleus $\left(I=\frac{3}{2}\right)$ of $\mathrm{LiBF}_{4}$ complexed to a liquid crystal containing four phenyl rings in the main core and attached polyethylene glycol moiety (courtesy of Dr. J. P. Bayle, Universite de Paris-Sud, Orsay, France) at the resonance frequency of $194.37 \mathrm{MHz}$ on a Bruker DRX-500 NMR spectrometer at $310 \mathrm{~K}$. This system exhibits a nematic phase from 305 to $332 \mathrm{~K}$. The energy level diagram of a spin $\frac{3}{2}$ system and the ${ }^{7} \mathrm{Li}$ NMR spectrum in the oriented phase are shown in Fig. 1. The spectrum has a quadrupolar splitting of $1.15 \mathrm{kHz}$ between the center tran- 
(a)

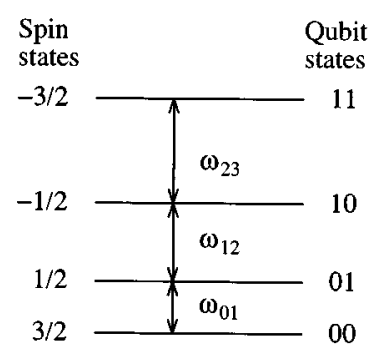

(b)

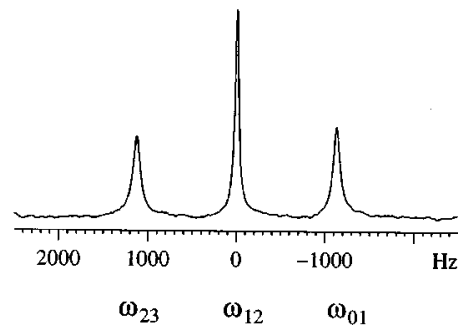

FIG. 1. (a) Schematic energy level diagram for a spin $\frac{3}{2}$ system and (b) equilibrium ${ }^{7} \mathrm{Li}$ NMR spectrum of $\mathrm{LiBF}_{4}$ oriented in a liquid crystalline matrix at $310 \mathrm{~K}$, obtained using a hard $\pi / 2$ pulse with a Bruker DRX-500 NMR spectrometer.

sition and the satellites at $310 \mathrm{~K}$. The following notation is used in our pulse schemes: $\chi(\alpha, \omega)$ is a selective pulse at frequency $\omega$, with a nutation angle $\alpha ; \chi(\alpha)$ is a nonselective hard pulse of nutation angle $\alpha ; G$ is a pulsed magnetic field $z$ gradient to kill transverse magnetization.

\section{CREATION OF PSEUDOPURE STATES}

An ensemble of nuclear spin systems with each member described by the same state vector is said to be in a pure state. $^{12,13}$ In a mixed state, on the other hand, all members cannot be described by the same state vector. In equilibrium at a finite temperature and magnetic field, an ensemble of nuclear spins will be in a mixed state, and can be described by a density matrix:

$$
\hat{\sigma}=c \hat{\mathbf{I}}+\hat{\sigma}_{\Delta},
$$

where $\hat{\mathbf{I}}$ is the identity and $\hat{\sigma}_{\Delta}$ is a traceless, deviation density matrix. The factor $c$ is very large $\left(\approx 10^{+6}\right)$ for nuclear spins at room temperature. The identity part does not evolve under rf pulses and system Hamiltonian, and also does not contribute to the measured signal. The entire signal arises from the deviation density matrix. At equilibrium $\sigma$ as well as $\sigma_{\Delta}$ are in a mixed state. While it is difficult to create $\sigma$ in a pure state (needs a very low temperature), the deviation density matrix can be made to behave as if it is in a pure state, by using rf pulses and magnetic field gradients. Such a system, where the deviation density matrix corresponds to a pure state, is known as a pseudopure state. Pseudopure states have been proposed and implemented using spin $\frac{1}{2}$ nuclei by many workers. ${ }^{7,12-19}$ Pseudopure states have also been implemented on a spin $\frac{3}{2}$ system, by using double-quantum pulses. ${ }^{4}$ Here we demonstrate the creation of pseudopure states in a spin $\frac{3}{2}$ system by using single-quantum pulses (Fig. 2). The energy levels in Fig. 2 are labeled, following Ref. 4, as 11, 10,01 , and 00 corresponding to the eigenstates of the system for $m_{z}=-\frac{3}{2},-\frac{1}{2}, \frac{1}{2}$, and $\frac{3}{2}$, respectively, and the deviation populations are represented by filled circles. The equilibrium deviation population and the spectrum obtained by using a single hard pulse of small angle $(\pi / 20)$ is shown in Fig. 2(a). The spectral intensities are given by $\left(P_{i}-P_{j}\right)$ multiplied by the square of the matrix elements of the ladder operators and for the spin $\frac{3}{2}$ case, they should be in the ratio 3:4:3 (experimental integrated intensities are in the ratio 1:1.6:1). The

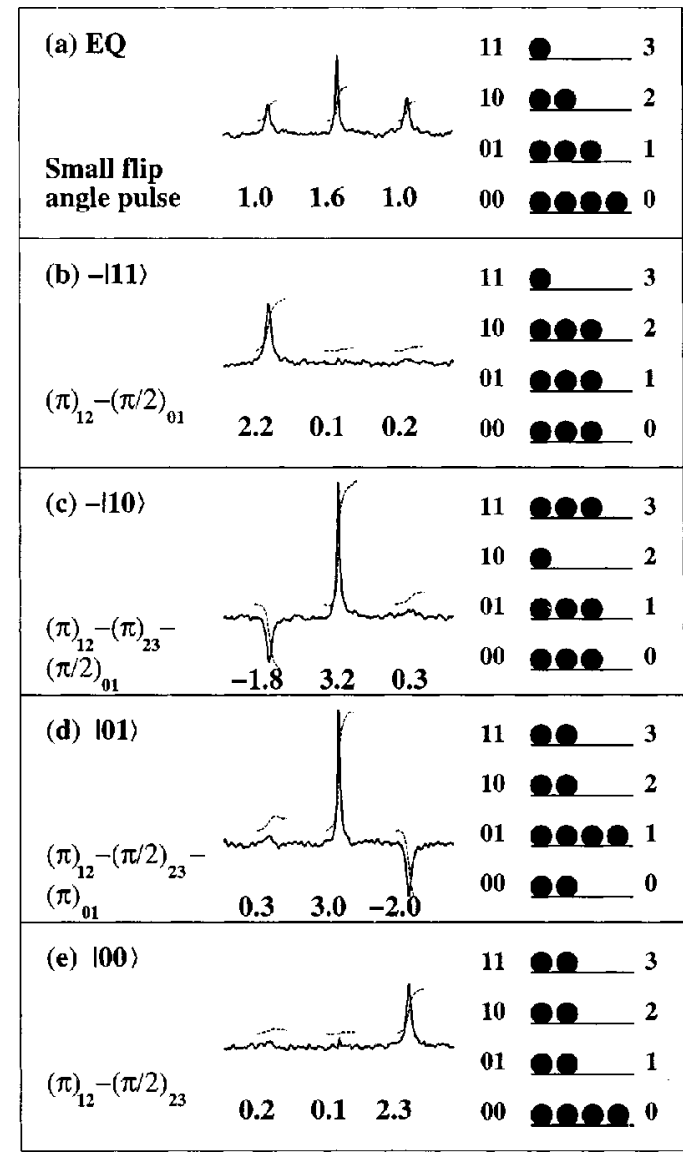

FIG. 2. Equilibrium spectrum (a) and spectra corresponding to different pseudopure states (b)-(e). All spectra are shown in the same scale. Integrated intensities of each peak are shown below each spectrum. The population distribution in each case is shown on the right-hand side. The pulse sequence used for the creation of the pseudopure state in each case is shown on the left-hand side. The lengths of rf $\pi$ pulses were $20 \mu$ s and $2 \mathrm{~ms}$, respectively, for the hard pulse and transition-selective pulse. Following these pulses, a $2 \mathrm{~ms}$ long sine-bell-shaped gradient pulse $\mathrm{G}$ of strength 25 $\mathrm{G} / \mathrm{cm}$ was used in each case to destroy the transverse magnetization. Following the gradient pulse, a rf detection pulse $(1 \mu \mathrm{s}$ long) corresponding to a small flip angle $(\pi / 20)$ was used. A total number of 16 scans were acquired in each case. For $\pi$ pulses a phase cycle of $(x,-x)$ was employed.

creation of pseudopure states for each of these four levels are shown in Figs. 2(b)-2(e), respectively, for 11, 10, 01, and 00 levels. The pseudopure state corresponding to level 11 is obtained by applying a transition-selective $\pi$ pulse on transition $1 \longrightarrow 2$, interchanging the populations of levels 1 and 2 , followed by a $(\pi / 2)$ pulse on $0 \rightarrow 1$ transition which creates a transverse magnetization of frequency $\omega_{01}$ and equalizes to an average value the population of levels 0 and 1 . The created transverse magnetization is destroyed by a gradient pulse. This cascade thus equalizes the populations of levels 0,1 , and 2 with the level 3 having less population (labeled as $-|11\rangle$ pseudopure state following Ref. 4) as confirmed by the experimental spectrum shown in Fig. 2(b). Similar pseudopure states corresponding to other eigenstates can be prepared by the cascade of pulses given in Fig. 2 along with the corresponding experimental NMR spectra. These spectra are similar to the ones obtained in Ref. 4, except that here we have utilized selective pulses on single-quantum transitions. 
TABLE I. Various logic gates with the corresponding Boolean algebra, truth table, pulse sequence, unitary operator, and circuit diagram.

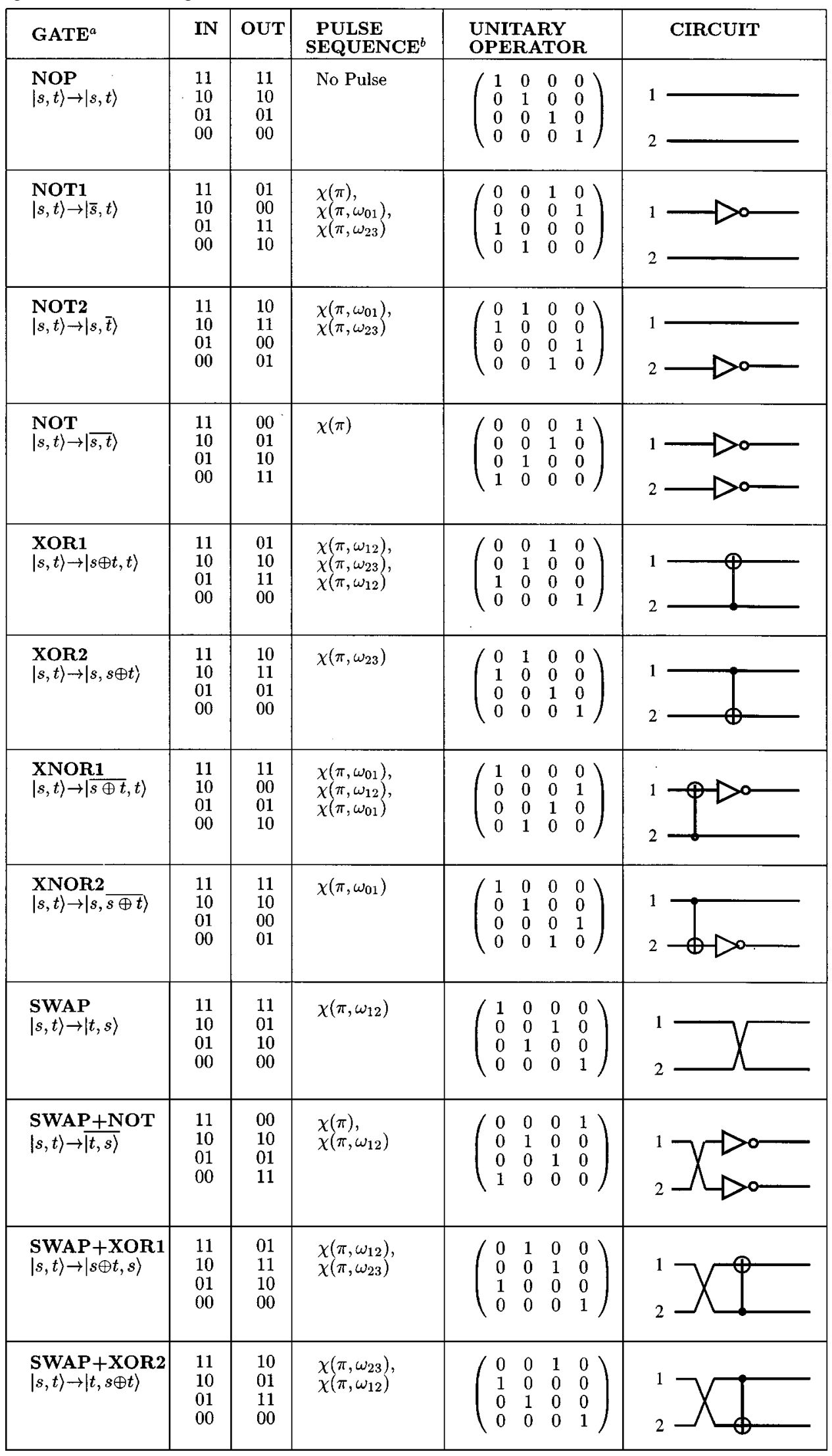


TABLE I. (Continued.)

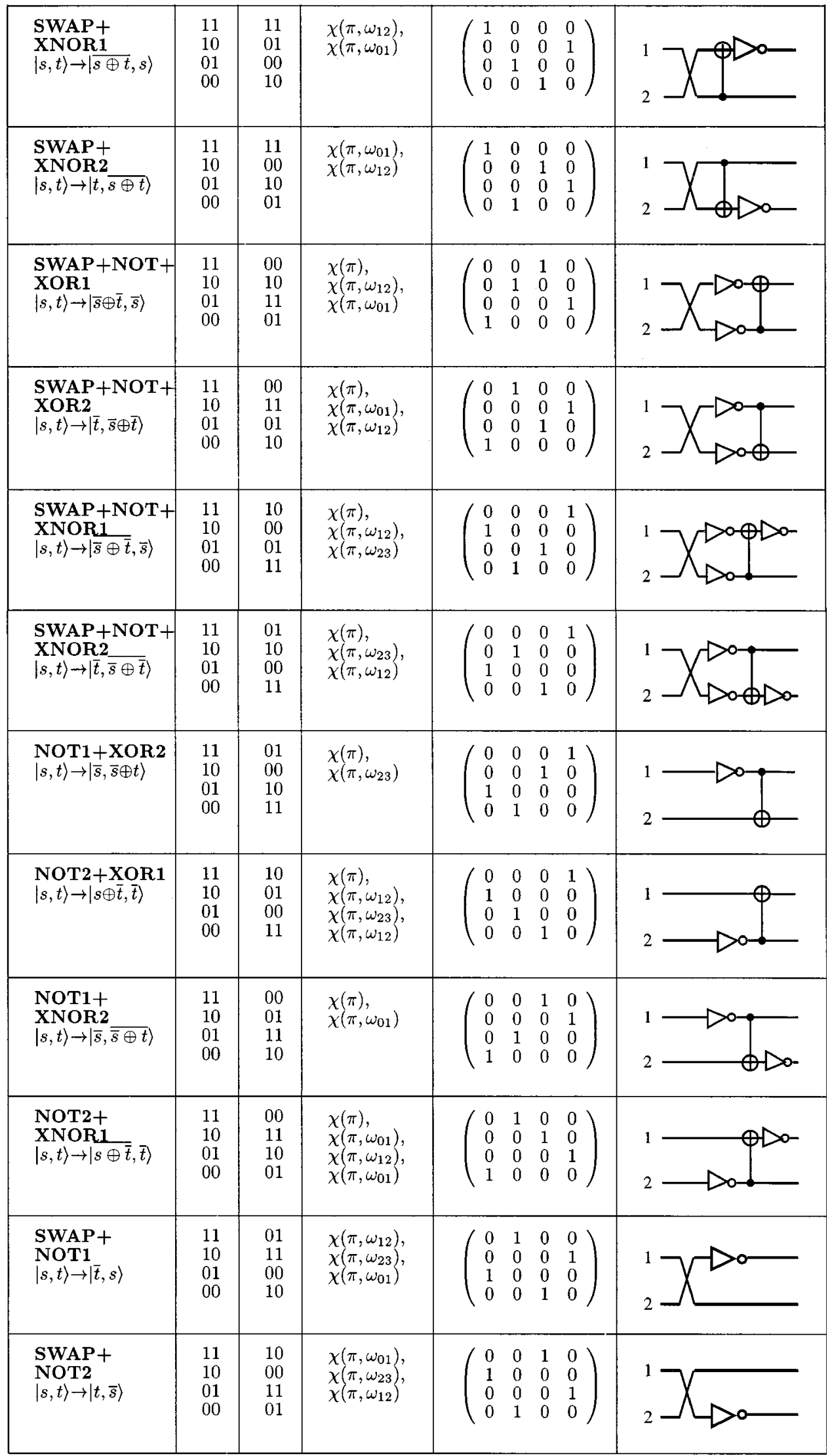

${ }^{\mathrm{a}}|s, t\rangle$ represents the state of the input qubits 1 and 2.

${ }^{\mathrm{b}}$ Order of pulses are important as all pulses do not commute. 


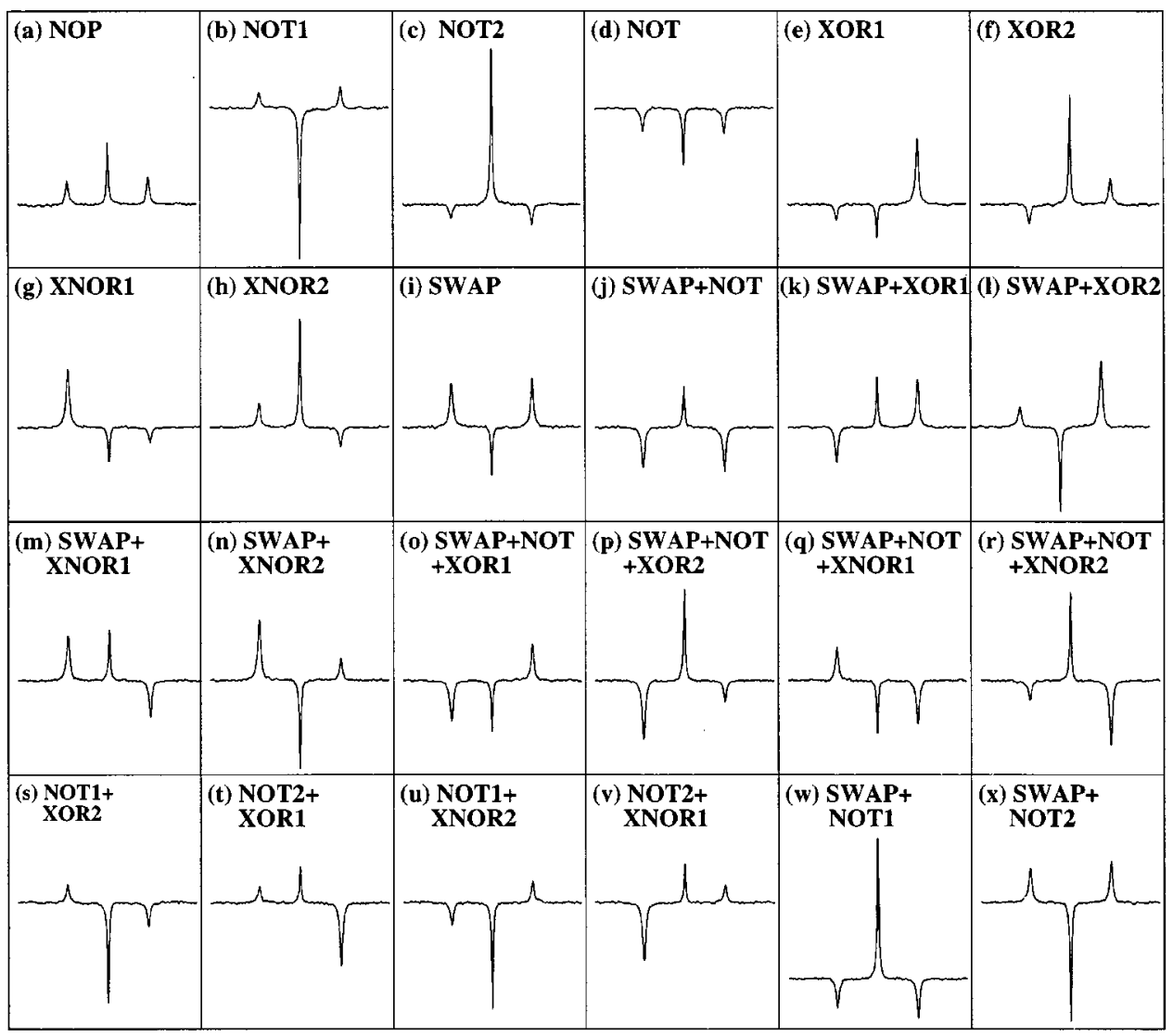

FIG. 3. ${ }^{7} \mathrm{Li}$ spectra corresponding 24 one-to-one logic gates plotted in the same intensity scale. The Boolean expression, truth table, and pulse sequence (except gradient and detection pulses) for each of these gates are given in Table I. For each $\pi$ pulse a phase cycle $(x,-x)$ was employed. A total number of 64 scans were collected for each spectrum with a recycle delay of $1.5 \mathrm{~s}$. The ratio of observed and expected (in brackets) integrated intensities are as follows: (a) 1.0:1.6:1.0 [1.0:1.3:1.0]; (b) 0.7:-3.8:0.6 [1.0:-3.9:1.0]; (c) -0.6:3.9:-0.9 [-1.0:3.9:-1.0]; (d) -1.0:-1.5:-1.1 [-1.0:-1.3:-1.0]; (e) $-0.7:-0.8: 2.6$ [-1.0:-1.3:3.0]; (f) $-0.8: 2.8: 0.9$ [-1.0:2.6:-1.0]; (g) 2.6:-0.9:-0.7 [2.0:-1.3:-1.0]; (h) 1.1:2.7:-0.9 [1.0:2.6:-1.0]; (i) 2.0:-1.1:1.9 [2.0:-1.3:2.0]; (j) $-1.8: 1.0:-1.9$ [-2.0:1.3:-2.0]; (k) -1.5:1.2:1.9 [-2.0:1.3:2.0]; (1) 1.0:-2.1:2.6 [1.0:-2.6:3.0]; (m) 2.0:1.3:-1.5 [2.0:1.3:-2.0]; (n) 2.7:-2.1:0.8 [3.0: $-2.6: 1.0]$; (o) -1.9:-1.3:1.4 [-2.0:-1.3:2.0]; (p) -2.6:2.2:-1.0 [-3.0:2.6:-1.0]; (q) 1.4:-1.3:-1.9 [2.0:-1.3:-2.0]; (r) -0.9:2.1:-2.6 [-1.0:2.6:-3.0]; (s) $0.8:-2.5:-1.1[1.0:-2.6:-1.0]$; (t) $0.7: 0.8:-2.7$ [1.0:1.3:-3.0]; (u) -1.0:-2.7:0.7 [-1.0:-2.6:1.0]; (v) -2.6:0.9:0.6 [-3.0:1.3:1.0]; (w) - 1.2:3.5:-1.7 [-2.0:3.9:-2.0]; (x) 1.5:-3.0:1.4 [2.0:-3.9:2.0].

Reference 4 utilized high-power pulses in the middle of two single-quantum transitions to excite the double-quantum coherence.

\section{IMPLEMENTATION OF NMR LOGIC GATES}

Several papers have appeared in the literature outlining the NMR implementation of logical gates by the use of oneand two-dimensional NMR up to five qubits. ${ }^{7-9,20-35}$ All these gates have been implemented on coupled spin $\frac{1}{2}$ nuclei in a solution state or in an oriented state. We demonstrate here the first implementation of a complete set of 24 one-toone 2-qubit logical gates on a spin $\frac{3}{2}$ nucleus, oriented in a liquid crystalline matrix, using one-dimensional NMR and single-quantum transition-selective $\pi$ pulses (Table I, Fig. 3). The pulse sequence used for the implementation of each gate is shown in the fourth column of Table I and all the output populations are measured by using a hard nonselective pulse of flip angle $\pi / 20$, preceded by a gradient pulse to destroy unwanted transverse magnetization. The NOP gate requires no computation pulse and yields the equilibrium spectrum. NOT2 (NOT on bit 2) requires two commuting transition-selective $\pi$ pulses on unconnected transitions.
NOT (NOT on both bits) is identical to the spin $\frac{1}{2}$ case and needs a non-selective $\pi$ pulse. The XOR1 (unlike the $\operatorname{spin} \frac{1}{2}$ case $^{7}$ ) requires a double-quantum $\pi$ pulse on transition $1 \rightarrow 3$, which is achieved here by a cascade of noncommuting $\pi$ pulses on $1 \rightarrow 2,2 \rightarrow 3$, and $1 \rightarrow 2$ transitions. XNOR1 requires inversions of levels 0 and 2, which are also separated by two quanta. The SWAP operation here is much simpler than the spin $\frac{1}{2}$ case $^{7}$ and needs only a selective $\pi$ pulse on transition $1 \rightarrow 2$. Like the spin $\frac{1}{2}$ case, several many-in-one (portmanteau) gates ${ }^{7}$ have also been implemented here, all needing selective or nonselective single-quantum $\pi$ pulses. The Boolean algebra, truth table, pulse sequence, unitary operator, and circuit diagram of each of these gates are shown in Table I, and the corresponding spectra in Fig. 3. The spectra corresponding to the 24 gates shown in Fig. 3 are distinct from each other and hence are characteristics of the gates.

\section{CONCLUSION}

In this paper we have demonstrated that by the use of transition-selective single-quantum pulses, it is possible to create pseudopure states and implement 2-qubit logical gates in a $\frac{3}{2}$ spin system oriented partially in a liquid crystal matrix. 
This is the first such implementation and extends the range of NMR for quantum computation. The increase of the number of qubits should now be possible by use of the higher spins (for example, $I=\frac{7}{2}$ ), and by dipolar coupling between various spins oriented in liquid crystalline matrices. These systems can be further utilized for the implementation of Deutsch-Jozsa ${ }^{36}$ and Grover's ${ }^{37}$ algorithms. Such attempts are continuing in our laboratory.

${ }^{1}$ J. A. Jones, quant-ph/0002085; prepared for the Fortschritte der Physik special issue, "Experimental proposals for quantum computation."

${ }^{2}$ C. S. Yannoni, M. H. Sherwood, D. C. Miller, I. L. Chuang, L. M. K. Vandersypen, and M. G. Kubinec, Appl. Phys. Lett. 75, 3563 (1999).

${ }^{3}$ M. Marjanska, I. L. Chuang, and M. G. Kubinec, J. Chem. Phys. 112, 5095 (2000)

${ }^{4}$ A. K. Khitrin and B. M. Fung, J. Chem. Phys. 112, 6963 (2000).

${ }^{5}$ P. Dhiel and C. L. Khetrapal, NMR—Basic Principles and Progress, Vol. 1 (Springer-Verlag, New York, 1969), pp. 1-95.

${ }^{6}$ K. Dorai and A. Kumar, J. Magn. Reson. 114, 155 (1995).

${ }^{7}$ K. Dorai, Arvind, and A. Kumar, Phys. Rev. A 61, 042306 (2000).

${ }^{8}$ T. S. Mahesh, K. Dorai, Arvind, and A. Kumar, J. Magn. Reson. 148, 95 (2001).

${ }^{9}$ K. Dorai, T. S. Mahesh, Arvind, and A. Kumar, Current Sci. 79, 1447 (2000).

${ }^{10}$ Arvind, K. Dorai, and A. Kumar, quant-ph/9909067.

${ }^{11}$ K. Dorai, Arvind, and A. Kumar, Phys. Rev. A (in press).

${ }^{12}$ N. Gershenfeld and I. L. Chuang, Science 275, 350 (1997).

${ }^{13}$ D. G. Cory, A. F. Fahmy, and T. F. Havel, Proc. Natl. Acad. Sci. U.S.A. 94, 1634 (1997).

${ }^{14}$ E. Knill, I. Chuang, and R. Laflamme, Phys. Rev. A 57, 3348 (1998).

${ }^{15}$ D. G. Cory, W. Maas, M. Price, E. Knill, R. Laflamme, W. H. Zurek, T. F. Havel, and S. S. Somaroo, Phys. Rev. Lett. 81, 2152 (1998).

${ }^{16}$ I. L. Chuang, N. Gershenfeld, M. G. Kubinec, and D. W. Leung, Proc. R. Soc. London, Ser. A 454, 447 (1998)
${ }^{17}$ L. M. K. Vandersypen, C. S. Yannoni, M. H. Sherwood, and I. L. Chuang, Phys. Rev. Lett. 83, 3085 (1999).

${ }^{18}$ U. Sakaguchi, H. Ozawa, and T. Fukumi, Phys. Rev. A 61, 042313 (2000).

${ }^{19}$ Y. Sharf, T. F. Havel, and D. G. Cory, Phys. Rev. A 62, 052314 (2000).

${ }^{20}$ A. Barenco, C. H. Bennett, R. Cleve, D. P. DiVincenzo, N. Margolus, P. Shor, T. Sleator, J. Smolin, and H. Weinfurter, Phys. Rev. A 52, 3457 (1995).

${ }^{21}$ V. Vedral, A. Barenco, and A. Ekert, Phys. Rev. A 54, 147 (1996).

${ }^{22}$ M. A. Nielson and I. L. Chuang, Phys. Rev. Lett. 79, 321 (1997).

${ }^{23}$ D. G. Cory, M. D. Price, and T. F. Havel, Physica D 120, 82 (1998).

${ }^{24}$ J. A. Jones, R. H. Hansen, and M. Mosca, J. Magn. Reson. 135, 353 (1998).

${ }^{25}$ Z. L. Madi, R. Bruschweiler, and R. R. Ernst, J. Chem. Phys. 109, 10603 (1998).

${ }^{26}$ V. Buzek, M. Hillery, and R. F. Werner, Phys. Rev. A 60, R2626 (1999).

${ }^{27}$ N. Linden, E. Kupce, and R. Freeman, Chem. Phys. Lett. 311, 321 (1999).

${ }^{28}$ M. D. Price, S. S. Somaroo, A. E. Dunlop, T. F. Havel, and D. G. Cory, Phys. Rev. A 60, 2777 (1999).

${ }^{29}$ E. Knill, R. Laflamme, R. Martinez, and C. H. Tseng, Nature (London) 404, 368 (2000)

${ }^{30}$ D. Leung, L. Vandersypen, X. Zhou, M. Sherwood, C. Yannoni, M. Kubinec, and I. Chuang, Phys. Rev. A 60, 1924 (1999).

${ }^{31}$ D. W. Leung, I. L. Chuang, F. Yamaguchi, and Y. Yamamoto, Phys. Rev. A 61, 042310 (2000).

${ }^{32}$ D. Collins, K. W. Kim, W. C. Holton, H. Sierzputowska-Gracz, and E. O. Stejskal, Phys. Rev. A 62, 022304 (2000).

${ }^{33}$ M. D. Price, T. F. Havel, and D. G. Cory, New J. Phys. 2, 10 (2000).

${ }^{34}$ R. Marx, A. F. Fahmy, J. M. Myers, W. Bermel, and S. J. Glaser, Phys. Rev. A 62, 012310 (2000).

${ }^{35}$ A. Muthukrishnan and C. R. Stroud, Jr., Phys. Rev. A 62, 052309 (2000).

${ }^{36}$ D. Deutsch and R. Jozsa, Proc. R. Soc. London, Ser. A 439, 553 (1992).

${ }^{37}$ L. K. Grover, Phys. Rev. Lett. 79, 325 (1997). 